\title{
Is Touch Gating due to Sensory or Cognitive Interference?
}

\author{
Daniel E. Harpera, ${ }^{*}$ and Mark Hollins ${ }^{a, b}$ \\ aDepartment of Psychology, University of North Carolina at Chapel Hill, Chapel Hill, NC 27599 \\ bDepartment of Endodontics, School of Dentistry, University of North Carolina at Chapel Hill, \\ Chapel Hill, NC 27599
}

\begin{abstract}
Touch gating, the attenuation of tactile sensitivity in the presence of pain, is a well-documented phenomenon but its mechanism is unknown. The ability of pain to capture attention suggests that touch gating may be an example of distraction; but the fact that pain raises tactile but not auditory thresholds argues that touch gating is a form of somatosensory interaction. Therefore, the present study was carried out to determine whether touch gating is the result of sensory or cognitive interference. Touch gating was repeatedly produced by delivering a co-localized painful heat stimulus $\left(45^{\circ} \mathrm{C}\right)$ during forced-choice measurements of vibration threshold on the palm. Noxious heat significantly increased thresholds compared to those measured at normal skin temperature, and this interference did not decline over the course of an extended series of experiments during which pain intensity significantly habituated. Touch gating was thus related to the constant physical intensity, rather than the changing subjective intensity, of the noxious stimulus. For comparison, a form of unambiguously cognitive interference, the Stroop effect, was also measured repeatedly; it declined significantly across sessions, unlike touch gating interference. Finally, touch gating was not correlated with measures of participants' distractibility, fear of pain, hypervigilance, or anxiety - variables previously found to influence pain on a cognitive level. Taken together, the results suggest that touch gating is a robust, stimulus-locked form of sensory interaction, rather than a transitory result of distraction or other cognitive processes.
\end{abstract}

\section{Keywords}

touch gating; interference; pain habituation; distraction; attention

\section{Introduction}

The term touch gating refers to the phenomenon in which noxious stimulation reduces tactile sensitivity. The discoverers of touch gating suggested that this interference with tactile processing is purely sensory, based on finding that pain reduces tactile thresholds only when the stimuli are presented in close proximity to one another and that pain does not alter auditory thresholds [2,5]. However, there is abundant evidence that pain can distract from optimal performance in a variety of tasks [20], and tactile evoked activity in the primary somatosensory cortex (S1) can be modulated by shifts in attention [30,44]. If the noxious

\footnotetext{
(C) 2012 International Association for the Study of Pain. Published by Elsevier B.V. All rights reserved. *Corresponding author. Tel.: +1 704996 0773; fax: +1 919962 2537, harperd@email.unc.edu. The authors have no conflicts of interest.

Publisher's Disclaimer: This is a PDF file of an unedited manuscript that has been accepted for publication. As a service to our customers we are providing this early version of the manuscript. The manuscript will undergo copyediting, typesetting, and review of the resulting proof before it is published in its final citable form. Please note that during the production process errors may be discovered which could affect the content, and all legal disclaimers that apply to the journal pertain.
} 
stimulus used in a touch gating experiment taxed the attentional resources of the brain and compromised the processing of tactile stimulation through distraction, the obtained touch gating would be, at least in part, the result of non-sensory mechanisms.

The present study was carried out to determine whether touch gating involves cognitive interference. This question was addressed in three ways.

First, we sought to determine whether pain's ability to gate tactile signals changes as the noxious inducing stimulus is repeatedly applied. Painful stimuli that are novel [20], unpredictable [12], and perceived as intense [8,19] are best suited to cause distraction. Thus, it is not surprising that distraction by pain is most pronounced at pain's onset or that it habituates with repeated presentations of a noxious stimulus $[13,14,15,49]$. Likewise, perceived pain intensity of a noxious stimulus can habituate over seconds or minutes $[10,17,23,27,29,31,39,40]$, and it can also habituate with repeated painful stimulations spanning days or weeks $[4,26,34,42,46]$. By repeatedly testing subjects, we were able to make multiple assessments of touch gating using a noxious stimulus that became less novel, more predictable, and less painful as the measurements progressed.

Even if distraction is not involved, touch gating might be expected to decline as a participant habituates to the noxious stimulus, given what is known about the physiological bases of touch gating and habituation. Specifically, evidence suggests that the locus of touch gating is in the brain, because pain does not affect tactile primary afferent transmission in the dorsal columns [11]. Long-term pain habituation, on the other hand, is thought to involve a decrease in pain signals in the spinal cord as a result of descending inhibition [4]. Taken together, these findings make it seem likely that touch gating would grow weaker as habituation occurs.

Second, we included a canonical measure of cognitive interference with which touch gating can be compared, the Stroop color-word task [45]. Previous research has shown that Stroop interference declines as subjects practice the task [16,18,22,35,36,45]. If touch gating similarly involves the use of cognitive control to overcome the interfering effect of pain on touch, then like Stroop interference, it should decline as subjects learn to engage cognitive mechanisms to overcome the interfering stimulus.

Third, we determined correlations between touch gating and psychological characteristics that could potentially increase pain's interference with vibrotactile detection through cognitive processes, including fear of pain, hypervigilance, anxiety, and everyday distractibility.

\section{Methods}

\subsection{Participants}

Thirty-one undergraduate students were enrolled in the study. Potential subjects were recruited by means of a flyer posted on campus. Of the enrolled subjects, 24 (12 males and 12 females) completed the study. Three subjects withdrew before study completion because of scheduling conflicts or a lack of interest in continuing. Four others were discontinued by the experimenters, three for not following instructions and one because she rated the thermal stimulus as "100" (corresponding to "the most intense pain imaginable") during Session 1. Data from the seven enrolled participants who did not complete the study were not included in the analysis. Age of participants who completed the study ranged from 18 to 25 years $(\mathrm{M}=20.5 ; \mathrm{SD}=1.9)$. For each subject, the four sessions were conducted at the same time of day when possible. The mean number of days from one session to the next was 8.8 , and the 
median was 7 days. The study procedures were approved by the University's IRB, and written informed consent was obtained. Subjects were compensated for their participation.

\subsection{Apparatus}

2.2.1. Vibrotactile and thermal stimuli-Vibrations and thermal stimulation were applied using a custom-built Vibrotactile Laboratory System (VLS) (Dancer Design, 123 Boundary Road, St. Helens, Merseyside, WA10 2LU, England). This apparatus used a minishaker to produce vibrations of a circular, flat, aluminum contactor (diameter $=8 \mathrm{~mm}$ ) that was raised to make contact with the subject's skin through a circular hole (diameter $=$ $10 \mathrm{~mm}$ ) in a tabletop on which the subject's hand rested. The region immediately surrounding the hole consisted of an annular aluminum plate (outer diameter $=64 \mathrm{~mm}$ ). Water from a remote heating/refrigeration unit was pumped through thermally-insulated plastic tubing into the contactor and surrounding plate, in which hollowed-out canals permitted the flow of temperature-controlled water close to the surface on which the subject's hand rested. This method of temperature control was similar to that used by Apkarian et al. [2]. Vibrations were delivered to the thenar eminence of the right hand, and this and the surrounding region of skin were controlled to normal skin temperature $\left(32^{\circ} \mathrm{C}\right)$ or a painfully hot temperature $\left(45^{\circ} \mathrm{C}\right)$ depending on run type. Both the vibrations and the thermal stimulation were controlled by custom-designed LabView programs. A display monitor that was visible only to the experimenters was used to observe the progress of each run.

2.2.2. Color vision test-Because Stroop interference was measured in this study, it was necessary to ensure that all subjects had normal color vision. During the first session, color vision was tested using a set of pseudoisochromatic plates [1]. The test was administered to participants under lighting provided by a Macbeth easel lamp with a daylight filter. Based on the standard scoring method for this test [1], participants were required to miss no more than four of the plates to be eligible for continuing in the study. All participants passed the test.

2.2.3. Stroop stimuli-The stimuli designed to produce a Stroop effect were composed of five color words (Blue, Brown, Red, Green, and Yellow), which were presented in one of the four non-matching colors (ex. the word "Red" presented in blue) during interference runs. Each stimulus set consisted of 60 color words, 12 of each color, that were simultaneously displayed on a computer monitor in a 4-column, X-by-Y array. In the 12 occurrences of each word in the array, it appeared in each of the other four colors an equal number of times (i.e., 3 times). The order in which the words were arranged was pseudorandomly determined, subject to the constraint that a given color or a given word did not appear three or more times in succession.

For a baseline condition against which interference could be calculated, control stimulus sets were created that contained series of X's instead of words. The same constraints were placed on the development and randomization of these stimulus sets, including 12 presentations of each of the five colors and no more than two stimuli of the same color in succession. To control for the fact that the different length of the words may have affected the difficulty of the task in the interference condition, different numbers of X's were used in the stimuli of the control sets, corresponding to the length of the words used to produce interference. Therefore, of the 60 stimuli in a control set, XXX, XXXX, and XXXXXX appeared 12 times each (for the words Red, Blue, and Yellow, respectively) and XXXXX appeared 24 times (for Green and Brown).

Stroop interference (interference set time - control set time) was measured four times in each of the four sessions (16 times in all). Therefore 16 different interference sets and 16 
different control sets were used. The order in which they were presented to a given participant was randomly determined, subject to the constraint that each set was used one time per subject. For each measure, whether a control set preceded an interference set, or vice versa, was random.

2.2.4. Questionnaires-At the beginning of Sessions 2 and 4, subjects completed the Fear of Pain Questionnaire-III (FOPQ) [38], the Pennebaker Inventory of Limbic Languidness (PILL) [41], and the State portion of the State Trait Anxiety Inventory (SAI) [43]. The Cognitive Failures Questionnaire (CFQ) [6], a well-validated measure of everyday distractibility, was also administered; it contains questions such as "Do you fail to listen to people's names when you are meeting them?" We also obtained numerical ratings of any pain and unpleasantness experienced upon subjects' arrival at the laboratory and during the previous two weeks.

\subsection{Procedure}

2.3.1. Consent, color vision test, and Stroop task training-At the beginning of Session 1 the general procedures were explained and informed consent was obtained. The overhead lights were turned off, and a Macbeth easel lamp with a daylight filter was illuminated for the administration of the color vision test.

Next, the subject was instructed in the Stroop task procedures using a series of PowerPoint slides presented on a computer monitor. At the end of this training, the subject practiced the Stroop task briefly, using a short set of interference stimuli. Any mistakes were corrected by the experimenters.

2.3.2. Vibrotactile threshold training-The subject was seated at the VLS table and trained in the procedure for measuring vibrotactile threshold in the absence of any noxious thermal stimulation. Headphones producing pink noise were worn by subjects during all measurements of vibration threshold. The subject was instructed to place the palm of the right hand flat on the tabletop with the middle of the thenar positioned over the hole containing the vibrotactile contactor. The contactor was then raised until it lightly touched the subject's skin; this was made evident by a slight uptick on a force readout on the experimenter's computer monitor. Following light contact with the skin, the contactor was raised an additional $1 \mathrm{~mm}$ to provide stable contact between the hand and the minishaker.

Vibration threshold was measured at a frequency of $33 \mathrm{~Hz}$ using two-interval forced-choice (2-IFC) tracking. Each trial in the 2-IFC threshold determination contained two intervals (A and $\mathrm{B}$ ), each $1 \mathrm{~s}$ in duration, separated by a gap of $1 \mathrm{~s}$. One of the two intervals contained a vibration and the other did not, the subject's job being to correctly indicate which of the two intervals contained the vibration on each trial. A computer monitor positioned $1 \mathrm{~m}$ directly in front of the subject kept the subject on track during runs. The monitor flashed the words "Get Ready" 1s before each trial began. Then, the letter "A" flashed on the screen during interval A, and "B" during interval B. After the intervals, the monitor prompted the subject's response ("Respond Now"), and he or she indicated during which interval the vibration had occurred using a joystick on a response box held in the subject's left hand. After the subject gave a response, he or she was given feedback on the monitor indicating whether it was right or wrong.

Following this feedback, the computer program adjusted the amplitude of the vibration stimulus for the next trial, which began after 2s. Amplitude was increased if the subject responded incorrectly or decreased if a subject made three, not necessarily consecutive, correct responses; otherwise, it remained the same. Each run consisted of 40 trials. Vibration amplitude was adjusted in steps of $3 \mathrm{~dB}$ during the first 20 trials and $1.5 \mathrm{~dB}$ during the last 20 
trials to gain more precision during the latter half of the run. Threshold was defined as the mean log amplitude $(\mathrm{dB})$ of the last eight trials in a run. For most subjects, vibration amplitude started at $18 \mathrm{~dB}$ (re: $1 \mu \mathrm{m})$ on the first trial of a run; two subjects had unusually low thresholds, necessitating the use of a lower initial amplitude $(14 \mathrm{~dB})$ in their runs. Four measurements of vibration threshold were made during Session 1, while VLS temperature was held near normal skin temperature $\left(32^{\circ} \mathrm{C}\right)$, to ensure that subjects were well-trained in the task.

2.3.3. Stroop task-Following each measurement of vibration threshold, subjects were seated in front of a different computer, where the Stroop task was administered on a monitor positioned approximately $0.5 \mathrm{~m}$ in front of the subject. Following a countdown of "ready, set, go," a Stroop stimulus set appeared on the screen. The subject was instructed to report aloud the color of each stimulus in the set, progressing down each column, beginning with the leftmost column, as quickly as possible without making mistakes. Time to complete the set and the number of uncorrected mistakes, if any, were recorded. A stimulus set of the opposite type (i.e. interference or control) was administered after a delay of approximately 15s. After completing each measurement of Stroop interference, the subject left the experimental room for a 10-min break before starting the next vibrotactile threshold measurement.

2.3.4. Pain assessment-At the end of Session 1, following four measurements each of vibration threshold and Stroop interference, the subject underwent a pain assessment. Here the subject's task was to periodically (every 30 s) rate the intensity of a continuous thermal stimulus $\left(45^{\circ} \mathrm{C}\right)$, using a zero-to- 100 scale where zero meant "no pain" and 100 meant "the most intense pain imaginable". The temperature of the contactor and surround was initially adjusted to $40^{\circ} \mathrm{C}$, and after the subject's hand was in place stimulus temperature was gradually raised $(\sim 1.5 \mathrm{~min})$ to $45^{\circ} \mathrm{C}$. The subject verbally rated pain intensity at the outset of the run and again every $30 \mathrm{~s}$ for an overall duration of $4 \mathrm{~min}$.

In summary, during Session 1 subjects took the color vision test and completed a short Stroop training exercise. Four measurements of vibration threshold were made while the VLS was at a neutral temperature. Each measurement of vibration threshold was followed by a measurement of Stroop interference and a 10-min break. Following the fourth measurement set, the pain assessment was carried out.

If a subject passed the color vision test, followed instructions in the vibrotactile threshold and Stroop experiments, and found the noxious stimulus tolerable, he or she was invited to come back for Sessions 2-4.

2.3.5. Main Experiment-The main experiment (i.e. repeated measurements of touch gating) took place during three sessions (Sessions 2-4) that were separated by intervals of approximately one week. Questionnaires were administered at the beginning of Sessions 2 and 4. Similar to the experimental timeline for Session 1, each main experimental session entailed four measurements of vibration threshold, and each vibration threshold measurement was followed by a measurement of Stroop interference and a break.

2.3.5.1. Repeated gating group: For half of the subjects, constituting the repeated gating (RG) group ( $\mathrm{N}=12)$, measurements of vibrotactile thresholds in the presence of co-localized noxious heat (concurrent heat runs; CHRs) were obtained, in alternation with threshold measurements without heat (no-heat runs; NHRs). Touch gating magnitudes were determined by subtracting thresholds measured at normal skin temperature (NHRs) from the generally higher thresholds measured in the presence of noxious heat (CHRs). 
When measurements of vibrotactile threshold were made with concurrent noxious heat stimulation (CHRs), the temperature of the VLS was controlled to $40^{\circ} \mathrm{C}$ before the start of a run. During the first $1.5 \mathrm{~min}$, temperature increased gradually to $45^{\circ} \mathrm{C}$, as in the pain assessment protocol. Subjects reported pain intensity using the 0-100 scale at the outset and again every thirty seconds for $1.5 \mathrm{~min}$. The 2 -IFC program was initiated following the pain rating obtained $1.5 \mathrm{~min}$ into the run, using parameters identical to those used in Session 1 $(33 \mathrm{~Hz}, 40$ trials, steps of 3 followed by $1.5 \mathrm{~dB}$, etc.). To periodically monitor perceived pain during vibrotactile threshold determinations, the 2 -IFC program was paused after every $10^{\text {th }}$ trial (about once per minute) and a pain rating was obtained. This procedure continued until the completion of 40 trials, at which point a final pain rating was obtained before the run ended (See Figure 1A). Baseline measurements of vibrotactile sensitivity were provided by no-heat runs (NHRs), which were carried out in an identical fashion (including starting the 2-IFC after $1.5 \mathrm{~min}$ and recording pain ratings), except the VLS temperature was controlled to normal skin temperature $\left(32^{\circ} \mathrm{C}\right)$ throughout (See Figure 1B).

RG subjects underwent four runs each session, with CHRs and NHRs alternating. Whether a CHR came first or second was counterbalanced and remained the same for a given subject across sessions. Each run was followed by a measurement of Stroop interference, including administration of both an interference and a control set. Thus there were four measurements of Stroop interference per session. Approximately 20min elapsed between successive vibration threshold runs, allowing time for a determination of Stroop interference and a break.

Touch gating was calculated by subtracting an NHR threshold (dB) from the CHR threshold (dB) by which it was immediately followed (if the NHR was first) or preceded (if the CHR was first). These calculations provided two such measurements of touch gating per session, and six measurements total (See Figure 1D).

2.3.5.2. Limited gating group: In designing the study, we considered the possibility that repeated noxious thermal stimulation might produce cumulative changes in tactile sensitivity, unrelated to touch gating. Therefore, we ran a separate group of subjects who received the same amount of noxious thermal stimulation as the RG group, but not simultaneously with vibrotactile testing. In this "limited gating" (LG) group, most runs that included heat consisted of a vibrotactile threshold measurement carried out at normal skin temperature, followed by a period of noxious stimulation. Runs of this type were called subsequent heat runs (SHRs; see Figure 1C). Following the vibrotactile threshold measurement portion of an SHR, the subject removed his or her hand from the apparatus $(\sim 1 \mathrm{~min})$ while the VLS was heated to $40^{\circ} \mathrm{C}$. Then, he or she placed the hand back onto the apparatus, and the temperature gradually rose to $45^{\circ} \mathrm{C}$. During an SHR, the subject gave periodic ratings not only during the period of vibrotactile threshold measurement, but during the subsequent 6.5-min period of noxious heat stimulation as well.

The LG group underwent a measurement of touch gating (i.e. CHR vs. NHR) at the beginning of session 2 and at the end of session 4, but between these two measurements they never received concurrent heat and vibrotactile stimulation; instead, they underwent SHRs and NHRs in alternation. Touch gating was therefore limited to an initial and a final measurement, although the two groups of subjects received the same overall amount of noxious thermal stimulation (see Figure 1E). Stroop interference was measured after each run.

In all three types of runs (CHR, SHR, and NHR), subjects gave pain ratings at 1-min intervals during the period of vibrotactile testing. These ratings were usually substantial during CHRs, but negligible during SHRs and NHRs. 


\section{Results}

\subsection{Vibrotactile threshold training}

The results from the four measurements of vibrotactile threshold obtained during Session 1 were analyzed to determine whether performance was stable before touch gating was measured in the subsequent sessions. Mean thresholds were 8.2, 8.2, 8.1, and $7.5 \mathrm{~dB}$ re: $1 \mu \mathrm{m}$ during run numbers $1,2,3$, and 4 , respectively. A repeated-measures ANOVA showed that the effect of run number was not significant $[F(3,69)=.65, p=.59]$. Vibration thresholds were therefore consistent during training.

\subsection{Touch gating}

3.2.1. RG group-Six measurements of touch gating were made in RG subjects. See Figure 2. A $6 \times 2$ repeated-measures ANOVA was implemented to determine whether, across multiple measurements (Measurement Number) of touch gating, vibrotactile thresholds were elevated in the presence of concurrent pain (Run Type), and whether this elevation changed. The main effect of Run Type was significant $[F(1,11)=16.3, p=.002]$, indicating impairment in vibrotactile sensitivity during CHRs. The main effect of Measurement Number was not significant $[F(5,55)=.21, p=.96]$, meaning no change in vibrotactile sensitivity was evident across the multiple measures. The interaction between Measurement Number and Run Type was also not significant $[F(5,55)=.65, p=.66]$, revealing no change in touch gating across the six measures of it.

3.2.2. LG group-Because participants in the LG group did not undergo CHRs between the initial and final measurements of touch gating, the effect of pain on vibration threshold could not be tracked on a measure-to-measure basis. However, paired-samples t-tests were conducted, comparing NHR thresholds with the thresholds obtained in SHRs, to determine whether threshold rose in SHRs in anticipation of pain. These tests revealed no significant difference between NHR and SHR vibration thresholds ( $p>.05$ for comparisons of runs 3 and 4; 5 and 6; 7 and 8; and 9 and 10). See Figure 3. If subjects did come to expect pain on alternate (i.e. SHR) runs, this did not produce any impairment in vibrotactile sensitivity. Paired-samples t-tests comparing CHRs and NHRs did, however, show significant touch gating for these subjects in the initial $[t(11)=5.00, p<.001]$ and final $[t(11)=4.91, p<$. 001] measures.

Thus, the effect of touch gating was reliable over the course of the measures in RG and LG subjects, and no artifact resembling touch gating occurred when NHRs and SHRs were compared in LG subjects.

3.2.3. Initial and final gating differences between groups?-A $2 \times 2 \times 2$ mixedmodel ANOVA was used to determine whether gating differed between groups during the initial and final measures. Within-subject factors were Measurement Number (initial or final) and Run Type (CHR or NHR), while the between-subjects factor was Group. The main effects of Measurement Number and Group were not significant $[F(1,22)=1.39, p=$. 25 and $F(1,22)=.03, p=.86$, respectively], indicating no difference in sensitivity from the initial to the final measure and no difference in sensitivity between groups. The main effect of Run Type was significant [ $F(1,22)=47.1, p<.001]$, revealing robust touch gating. None of the interactions was significant ( $p>.10$ for all); thus, touch gating, and the degree to which it withstood repeated testing, was not different for the groups.

\subsection{Pain ratings}

Because the temperature of the VLS rose gradually from an innocuous warm temperature $\left(40^{\circ} \mathrm{C}\right)$ to $45^{\circ} \mathrm{C}$ during the first $1.5 \mathrm{~min}$ of heat runs, pain ratings obtained during this time 
were omitted from analysis. A mixed-model ANOVA of ratings obtained once the VLS was at the target temperature (ratings 5-8) was employed to measure changes in perceived pain: Within-subject factors assessed changes in pain across sessions 2-4 (Session), between the first and second pain runs of each session (Run), and across the four ratings within each run (Rating). To determine whether these changes in perceived pain were different between RG and LG groups, Group was included as a between-subjects factor.

The significant effect of Rating $[F(3,66)=39.9, p<.001]$ indicates that pain increased (i.e. sensitized) within runs. See Figure 4. Across runs and sessions, however, the opposite (i.e. habituation) occurred. The main effect of $\operatorname{Run}[F(1,22)=7.3, p=.01]$ shows that pain significantly habituated between the first and second pain exposures within sessions, and a main effect of Session $[F(2,44)=10.9, p<.001]$ reveals significant pain habituation across sessions. See Figure 5. The lack of a main effect of $\operatorname{Group}[F(1,22)=1.3, p=.26]$ indicates no difference in pain sensitivity between RG and LG subjects. The only significant interaction was between Session, Rating, and Group $[F(6,132)=2.4, p=.03]$; inspection of the data indicated that for RG subjects, sensitization within runs tended to decrease across sessions, while for LG subjects this sensitization increased.

\subsection{Analysis of data pooled across groups}

3.4.1. Stroop interference-Since four Stroop interference runs were carried out within each session, there were 16 measurements of Stroop interference in all. See Figure 6. A $4 \times$ $4 \times 2$ repeated measures ANOVA, with Session (1-4), Run (1-4), and Stimulus Type (interference or control) was used to examine these data. The main effect of Stimulus Type revealed robust interference $[F(1,23)=144.0, p<.001]$. The main effect of Session was significant $[F(3,69)=34.9, p<.001]$ but the effect of $R$ un was not $[F(3,69)=0.9, p=.46]$, indicating decreasing times for both interference and control sets across, but not within, sessions. The Session $\times$ Run interaction was significant $[F(9,207)=4.9, p<.001]$; although there was no main effect of Run, improvement within sessions was reduced in later sessions. The Session $\times$ Stimulus Type interaction was significant $[F(3,69)=7.4, p<.001]$, revealing a reduction in Stroop interference across sessions. See Figure 7. No other interaction was significant.

3.4.2. Touch gating correlations with other variables-Psychosocial questionnaires measuring fear of pain (FOPQ), distractibility (CFQ), hypervigilance (PILL), and state anxiety (SAI), as well as a questionnaire measuring current and recent pain, were administered to determine whether these psychological factors were related to touch gating magnitude. Pearson correlations between these questionnaire measures and initial and final measures of touch gating revealed that none was related to the amount of touch gating that occurred $(p>.10$ for all). See Table 1.

A Pearson correlation between initial touch gating and the first measure of Stroop interference (measure 1) revealed no relationship between these two types of interference $[r$ $=-.27, p=.20 \mathrm{]}$ at the outset of testing them. Likewise, a comparison of the two tasks following substantial practice on them showed that final touch gating was not correlated with the last measurement (measure 16) of Stroop interference $[r=-.04, p=.85]$.

Finally, touch gating was compared with perceived pain ratings, to determine whether subjective pain intensity is related to touch gating magnitude. There was no relationship between touch gating and perceived pain during the respective pain runs for the initial $[r=$ $-.12, p=.59]$ or final $[r=.07, p=.76]$ measures. 


\section{Discussion}

Three main lines of evidence point to touch gating being a sensory interaction between nociceptive and tactile signals, rather than a result of distraction or other high-level processes. First, touch gating was unchanged by repeated noxious stimulation, in spite of the fact that perceived pain habituated significantly over the same series of runs. Second, the amount of practice that subjects received in detecting vibrations in the presence of concurrent painful stimulation did not affect the amount of touch gating they showed. This resistance to change distinguishes touch gating from cognitive (e.g. Stroop) interference, which subjects gradually learned to overcome. Finally, touch gating magnitude was not related to any of the psychological variables measured by questionnaire in this study, suggesting that touch gating is not mediated by anxiety, distraction, hypervigilance, or fear of pain. These lines of evidence are discussed more fully in the following sections.

\subsection{Distraction and pain habituation}

If touch gating were the result of distraction by pain, then perceived pain should have been related to touch gating magnitude and touch gating should have habituated with pain. Intense chronic pain causes greater distraction than chronic pain of lower intensity [19]. Moreover, moment-to-moment changes in the painfulness of a constant thermal stimulus predict the magnitude of pain's distraction from a cognitive task on a trial-to-trial basis [8]. The fact that reported pain intensity was not correlated with touch gating magnitude in the present study shows that touch gating was not a function of the subjective experience of pain, and thus argues that it was not the result of distraction.

Furthermore, pain's ability to distract habituates $[13,14,15,49]$, presumably as the noxious stimulus loses its novelty and threat value [20,50]; and pain causes less distraction when it is predictable than when it is not [12]. Although our subjects did not explicitly report the perceived novelty or predictability of the noxious stimulus, repeated presentations of the same stimulus (in terms of temperature, rise time, stimulated area, and duration) spanning multiple runs within and across sessions became very familiar to subjects by the time of the final touch gating measurement. Subjects also learned that the thermal stimulus, while painful, did not cause any lasting discomfort or injury and was therefore not threatening. All of these cognitive changes likely reduced pain's ability to capture attention and serve as a distraction. The fact that touch gating magnitude nevertheless remained relatively constant throughout the experimental sessions therefore suggests that distraction is not the instrument with which pain gated touch in the present study.

Pain that is intense, novel, unpredictable, or threatening might increase vibrotactile threshold through distraction, and thus add to measured touch gating, but under everyday conditions (and those in the present study), touch gating is a form of sensory interaction that appears relatively immune to distraction.

\subsection{Cognitive interference}

To perform optimally in the Stroop task, subjects must effectively inhibit an automatic tendency to read interfering words [36] by engaging cognitive control mechanisms [9]. Similarly, to optimally detect vibrations in the presence of pain, subjects are required to ignore a stimulus that, given the proper parameters, can automatically enter attention [20]. In the present study, measurements of touch gating and Stroop interference both included a nointerference condition and an interference condition, and in both cases the interference condition was found to significantly hamper optimal performance. However, in terms of the results of these measures of interference, the parallels went no further. Touch gating magnitude was not correlated with Stroop interference, suggesting that the two types of 
interference are processed in different ways. In addition, this absence of a relationship implies that the degree of pain's detrimental effect on tactile processing is not a function of one's ability, or lack thereof, to effectively ignore stimuli through cognitive control.

Furthermore, there were striking differences in the way these two types of interference withstood repeated testing: Stroop interference declined significantly, as expected from earlier studies $[16,18,22,35,36,45]$, but touch gating remained robust. The reduction in Stroop interference with practice shows that subjects successfully learned cognitive strategies to cope with the interfering stimuli. In contrast, repeated practice detecting vibrations with concurrent pain was not successful in reducing pain's interference with touch, indicating that the two types of interference are fundamentally different.

\subsection{Psychological characteristics}

Additional support for the conclusion that touch gating is a sensory phenomenon comes from the lack of any observed correlations between touch gating and psychological variables that might be expected to influence a cognitive process. High somatic awareness (i.e. hypervigilance) has been shown to enhance pain's interference with cognitive tasks [21], but pain's effect on tactile sensitivity did not vary as a function of this psychological variable, nor was touch gating related to an individual's fear of pain or current anxiety. Likewise, scores on the CFQ, a measure of everyday distractibility, were unrelated to touch gating magnitude, although previous studies have reported correlations between CFQ scores and performance on a variety of cognitive tasks [7,24,25,28,32,33,37,47]. Touch gating therefore appears to be a relatively automatic process that is impervious to "top-down" influences.

\subsection{The physiology of pain habituation and touch gating}

The finding that pain habituation did not reduce the magnitude of the nociceptive system's inhibitory influence on touch is in agreement with neurophysiological and neuroimaging studies of pain habituation and touch gating. Bingel et al. [4] found that repeated daily pain stimulations caused decreases in perceived pain and pain-evoked activity in many regions of the pain system (e.g. insula, thalamus, secondary somatosensory cortex), but not in S1. In terms of pain habituation on a shorter timescale, repeated presentation of a thermal stimulus that was moved to fresh skin between trials (thus avoiding peripheral adaptation) caused pain ratings to significantly habituate, but pain-evoked activity in S1 was not reduced by the same magnitude [27]. These results suggest that central pain habituation may occur in cortical regions devoted to higher-level processing of pain, while leaving pain's influence on S1 relatively intact.

Several studies have implicated S1 as a possible locus of touch gating [3,48,51]. Under this hypothesis, nociceptive signals would have to reach $\mathrm{S} 1$ with unchanging intensity during habituation, in order to gate touch with unchanging magnitude (as the present study found). Alternatively, touch gating might occur at a lower level than S1, consistent with the fact that nociceptive signaling can alter the processing of tactile signals in the dorsal column nuclei [11].

Whether the critical mechanism resides in brainstem or cortex, there is an emerging consensus that touch gating occurs in the brain. In contrast, long-term pain habituation is accompanied by activation in areas associated with descending pain inhibition [4], and is therefore thought to suppress pain signals, at least in part, at the level of the spinal cord. Taken together, these results suggest that pain signals habituate at an earlier level than touch gating. The present results are, however, incompatible with this view, in that a decrease in the pain signal (long-term pain habituation) did not weaken an effect (touch gating) that this pain signal produces. 
One way to resolve this seeming contradiction is to hypothesize that pain habituation dampens signals that are responsible for perceived pain while leaving parallel signals that are responsible for closing the touch gate relatively intact. This interesting possibility warrants future investigation.

\subsection{Conclusions}

The present study employed a combination of approaches to increase understanding of touch gating, a phenomenon in which tactile sensitivity is impaired by the simultaneous presentation of a noxious stimulus. A key question was whether touch gating is more appropriately regarded as a form of sensory, or of cognitive, interference. Three lines of evidence were marshaled to answer this question. (1) Touch gating remained strong despite a significant decline, due to habituation, in the painfulness of the noxious stimulus that induced it. (2) Repeated activation of the touch gate did not weaken it; this stands in contrast to the reduction in cognitive (Stroop) interference that was observed in the same subjects. (3) Finally, touch gating magnitude was not related to distractibility or other psychological variables known to affect pain's ability to interfere with performance on various tasks. While extreme conditions (e.g. inducing fear in subjects) might add a top-down element (such as distraction) to measurements of touch gating, the evidence of the present study indicates that at its core, touch gating is a robust and relatively inflexible form of interaction between somatosensory submodalities, isolated from cognitive influences and dependent on afferent signals rather than on the subjective experiences that result from them.

\section{Acknowledgments}

We would like to thank Caitlin Murray and Edward McLaurin for their help in carrying out these experiments. This research was supported by NINDS grant NS045685.

\section{References}

1. American Optical Corporation. Pseudo-isochromatic plates for testing color perception. Philadelphia: The Beck Engraving Co., Inc; 1965.

2. Apkarian AV, Stea RA, Bolanowski SJ. Heat-induced pain diminishes vibrotactile perception: A touch gate. Somatosens Mot Res. 1994; 11:259-267. [PubMed: 7887057]

3. Apkarian AV, Stea RA, Manglos SH, Szeverenyi NM, King RB, Thomas FD. Persistent pain inhibits contralateral somatosensory cortical activity in humans. Neurosci Lett. 1992; 140:141-47. [PubMed: 1501770]

4. Bingel U, Schoell E, Herken W, Büchel C, May A. Habituation to painful nociceptive stimulation involves the antinociceptive system. Pain. 2007; 131:21-30. [PubMed: 17258858]

5. Bolanowski SJ, Maxfield LM, Gescheider GA, Apkarian AV. The effects of stimulus location on the gating of touch by heat- and cold-induced pain. Somatosens Mot Res. 2000; 17:195-204. [PubMed: 10895889]

6. Broadbent DE, Cooper PF, FitzGerald P, Parkes KR. The Cognitive Failures Questionnaire (CFQ) and its correlates. Brit J Clin Psychol. 1982; 21:1-16. [PubMed: 7126941]

7. Broadbent DE, Broadbent MHP, Jones JJ. Performance correlates of self-reported cognitive failure and obsessionality. Brit J Clin Psychol. 1986; 25:285-99. [PubMed: 3801732]

8. Buhle J, Wager TD. Performance-dependent inhibition of pain by an executive working memory task. Pain. 2010; 149:19-26. [PubMed: 20129735]

9. Carter CS, van Veen V. Anterior cingulate cortex and conflict detection: An update of theory and data. Cogn Affect Behav Neurosci. 2007; 7:367-79. [PubMed: 18189010]

10. Condes-Lara M, Calvo JM, Fernandez-Guardiola A. Habituation to bearable experimental pain elicited by tooth pulp electrical stimulation. Pain. 1981; 11:185-200. [PubMed: 7322602]

11. Costa-Garcia M, Nuñez A. Nociceptive stimuli induce changes in somatosensory responses of rat dorsal column nuclei neurons. Brain Res. 2004; 1025:169-76. [PubMed: 15464757] 
12. Crombez G, Baeyens F, Eelen P. Sensory and temporal information about impending pain: the influence of predictability on pain. Behav Res Ther. 1994; 32:611-22. [PubMed: 8085989]

13. Crombez G, Eccleston C, Baeyens F, Eelen P. The disruptive nature of pain: An experimental investigation. Behav Res Ther. 1996; 34:911-18. [PubMed: 8990542]

14. Crombez G, Eccleston C, Baeyens F, Eelen P. Habituation and the interference of pain with task performance. Pain. 1997; 70:149-54. [PubMed: 9150288]

15. Crombez G, Eccleston C, Baeyens F, Eelen P. When somatic information threatens, catastrophic thinking enhances attentional interference. Pain. 1998; 75:187-98. [PubMed: 9583754]

16. Davidson DJ, Zacks RT, Williams CC. Stroop interference, practice, and aging. Neuropsychol Dev Cogn B Aging Neuropsychol Cogn. 2007; 10:85-98. [PubMed: 17203134]

17. Defrin R, Pope G, Davis KD. Interactions between spatial summation, 2-point discrimination and habituation of heat pain. Eur J Pain. 2008; 12:900-9. [PubMed: 18280188]

18. Dulaney CL, Rogers WA. Mechanisms underlying reduction in Stroop interference with practice for young and old adults. J Exp Psychol. 1994; 20:470-84.

19. Eccleston C. Chronic pain and attention: A cognitive approach. Brit J Clin Psychol. 1994; 33:53547. [PubMed: 7874045]

20. Eccleston C, Crombez G. Pain demands attention: A cognitive-affective model of the interruptive function of pain. Psychol Bull. 1999; 125:356-66. [PubMed: 10349356]

21. Eccleston C, Crombez G, Aldrich S, Stannard C. Attention and somatic awareness in chronic pain. Pain. 1997; 72:209-15. [PubMed: 9272805]

22. Edwards S, Brice C, Craig C, Penri-Jones R. Effects of caffeine, practice, and mode of presentation on Stroop task performance. Pharmacol Biochem Be. 1996; 54:309-15.

23. Ernst M, Lee MHM, Dworkin B, Zaretsky HH. Pain perception decrement produced through repeated stimulation. Pain. 1986; 26:221-31. [PubMed: 3763235]

24. Forster S, Lavie N. High perceptual load makes everybody equal: Eliminating individual differences in distractibility with load. Psychol Sci. 2007; 18:377-81. [PubMed: 17576274]

25. Goldhammer F, Moosbrugger H, Krawietz SA. Fact-2 - The Frankfurt Adaptive Concentration Test: Convergent validity with self-reported cognitive failures. Eur J Psych Assess. 2009; 25:7382.

26. Greenspan JD, McGillis SLB. Thresholds for the perception of pressure, sharpness, and mechanically evoked cutaneous pain: Effects of laterality and repeated testing. Somatosens Mot Res. 1994; 11:311-7. [PubMed: 7778408]

27. Greffrath W, Baumgärtner U, Treede R. Peripheral and central components of habituation of heat pain perception and evoked potentials in humans. Pain. 2007; 132:301-11. [PubMed: 17533117]

28. Harris JE, Wilkins AJ. Remembering to do things: A theoretical framework and an illustrative experiment. Hum Learn. 1982; 1:123-36.

29. Hollins M, Harper D, Maixner W. Changes in pain from a repetitive thermal stimulus: The roles of adaptation and sensitization. Pain. 2011; 152:1583-90. [PubMed: 21454015]

30. Johansen-Berg H, Christensen V, Woolrich M, Matthews PM. Attention to touch modulates activity in both primary and secondary somatosensory areas. NeuroReport. 2000; 27:1237-41. [PubMed: 10817599]

31. Kleinböhl D, Trojan J, Konrad C, Hölzl R. Sensitization and habituation of AMH and C-fiber related percepts of repetitive radiant heat stimulation. Clin Neurophysiol. 2006; 117:118-30. [PubMed: 16256426]

32. Kanai R, Dong MY, Bahrami B, Rees G. Distractibility in daily life is reflected in the structure and function of human parietal cortex. J Neurosci. 2011; 31:6620-26. [PubMed: 21543590]

33. Kramer AF, Humphrey DG, Larish JF, Logan GD, Strayer DL. Aging and inhibition: Beyond a unitary view of inhibitory processing in attention. Psychol Aging. 1994; 9:491-512. [PubMed: 7893421]

34. Labus JS, Naliboff BD, Berman SM, Suyenobu B, Vianna EP, Tillisch K, Mayer EA. Brain networks underlying perceptual habituation to repeated aversive visceral stimuli in patients with irritable bowel syndrome. NeuroImage. 2009; 47:952-60. [PubMed: 19501173] 
35. MacLeod CM. Training on integrated versus separated Stroop tasks: The progression of interference and facilitation. Mem Cognition. 1998; 26:201-11.

36. MacLeod CM, Dunbar K. Training and Stroop-like interference: Evidence for a continuum of automaticity. J Exp Psychol. 1988; 14:126-35.

37. Martin M, Jones GV. Distribution of attention in cognitive failure. Hum Learn. 1983; 2:221-26.

38. McNeil DW, Rainwater AJ. Development of the Fear of Pain Questionnaire-III. J Behav Med. 1998; 21:389-410. [PubMed: 9789168]

39. Milne RJ, Kay NE, Irwin RJ. Habituation to repeated painful and non-painful cutaneous stimuli: a quantitative psychophysical study. Exp Brain Res. 1991; 87:438-44. [PubMed: 1769394]

40. Mobascher A, Brinkmeyer J, Warbrick T, Musso F, Schlemper V, Wittsack HJ, Saleh A, Schnitzler A, Winterer G. Brain activation patterns underlying fast habituation to painful laser stimuli. Int J Psychophysiol. 2010; 75:16-24. [PubMed: 19833154]

41. Pennebaker, JW. The psychology of physical symptoms. New York (NY): Springer-Verlag; 1982.

42. Rennefield C, Wiech K, Schoell ED, Lorenz J, Bingel U. Habituation to pain: Further support for a central component. Pain. 2010; 148:503-8. [PubMed: 20097005]

43. Spielberger, CD. Manual for the State-Trait Anxiety Inventory (STAI). Palo Alto, California: Consulting Psychologists Press; 1983.

44. Sterr A, Shen S, Zaman A, Roberts N, Szameitat A. Activation of SI is modulated by attention: a random effects fMRI study using mechanical stimuli. Neuroreport. 2007; 18:607-11. [PubMed: 17413666]

45. Stroop JR. Studies of interference in serial verbal reactions. J Exp Psychol. 1935; 18:643-62.

46. Taylor DJ, McGillis SLB, Greenspan JD. Body site variation of heat pain sensitivity. Somatosens Mot Res. 1993; 10:455-65. [PubMed: 8310782]

47. Tipper SP, Baylis GC. Individual differences in selective attention: The relation of priming and interference to cognitive failure. Person Individ Diff. 1987; 8:667-75.

48. Tommerdahl M, Delemos KA, Vierck CJ Jr, Favorov OV, Whitsel BL. Anterior parietal cortical response to tactile and skin-heating stimuli applied to the same skin site. J Neurophysiol. 1996; 75:2662-70. [PubMed: 8793772]

49. Vancleef LMG, Peters ML. Pain catastrophizing, but not injury/illness sensitivity or anxiety sensitivity, enhances attentional disruption by pain. J Pain. 2006; 7:23-30. [PubMed: 16414550]

50. Vancleef LMG, Peters ML. The interruptive effect of pain on attention. J Pain. 2006; 7:21-2. [PubMed: 16414549]

51. Whitsel BL, Favorov OV, Li Y, Lee J, Quibrera PM, Tommerdahl M. Nociceptive afferent activity alters the SI RA neuron response to mechanical skin stimulation. Cereb Cortex. 2010; 20:2900-15. [PubMed: 20308203] 


\section{A}
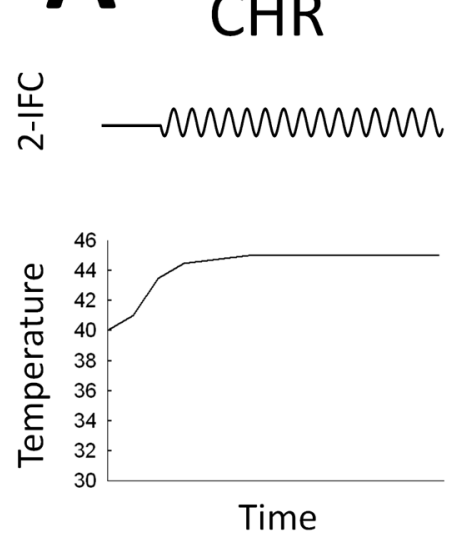

B
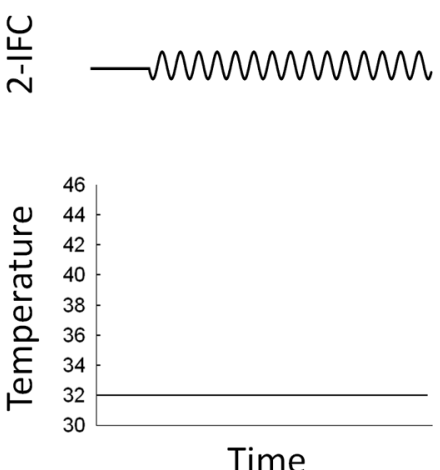
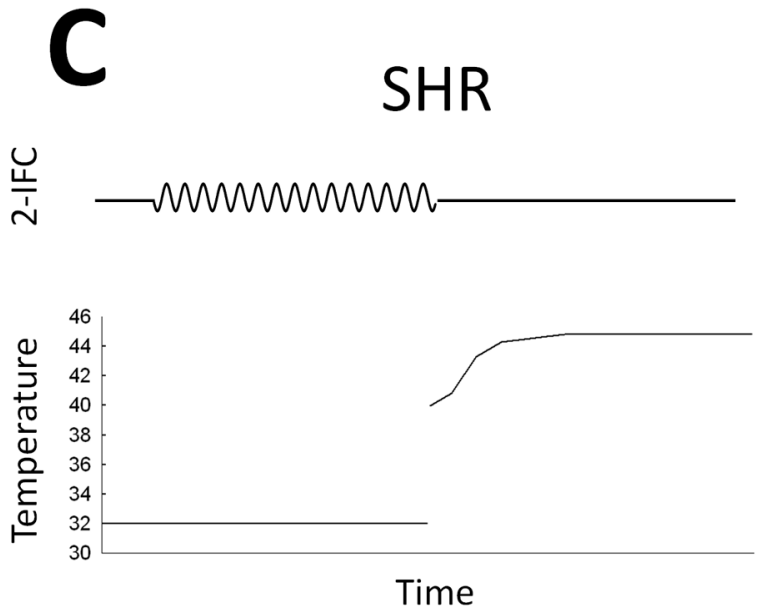

\section{TG Initial}
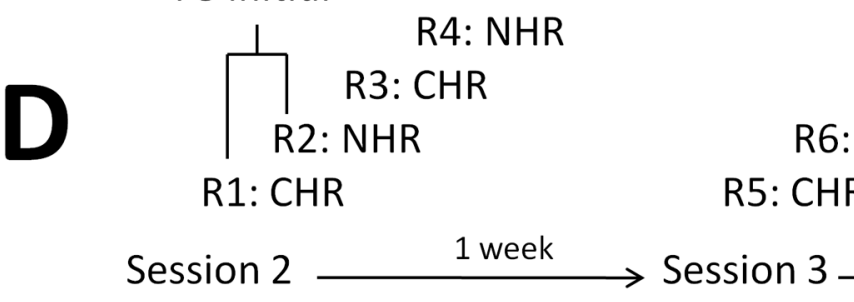

R8: NHR

R12: NHR

R7: CHR

R6: NHR

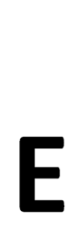

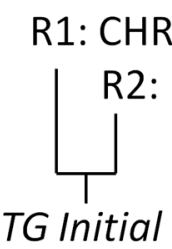

R5: SHR

R6: NHR

R9: SHR

\section{R7: SHR \\ R8: NHR}

R9: CHR

R3: SHR

R4: NHR

Figure 1.

Run types and main experiment timeline. The three run types: A) concurrent heat run (CHR), B) no heat run (NHR), and C) subsequent heat run (SHR), are pictured. The 2-IFC row indicates when a subject's hand was positioned on the VLS. No vibrations were presented for the first $1.5 \mathrm{~min}$ of each run (straight lines), after which vibration threshold was measured using the 2-IFC protocol (wavy lines). The temperature graphs indicate the temperature of the contactor and surround during each run type. Heat was presented concurrently with vibrotactile measurements only during CHRs. SHRs included a pain stimulus of the same intensity $\left(45^{\circ} \mathrm{C}\right)$ and duration $(6.5 \mathrm{~min})$ as that of CHRs, but it was presented after measurement of vibration threshold. D) Order of run types for a subject in the repeated gating (RG) group. E) Order of run types for a limited gating (LG) group subject. An initial measure of touch gating ( $T G$ Initial) was calculated for both groups of subjects using runs 1 and 2. Treatment of the groups differed until a final measurement of touch gating ( TG Final) was made (runs 11 and 12). Between initial and final measurements, RG subjects continued to undergo CHRs alternating with NHRs, while LG subjects underwent SHRs between NHRs. Run order was counterbalanced within each group so that half of the subjects started with a CHR (as shown in the figure) and the other half started with an NHR followed by a CHR (not shown). Following each run, a measurement of Stroop interference was made, including the control and interference conditions (not shown). Twenty minutes elapsed between runs within a session. 


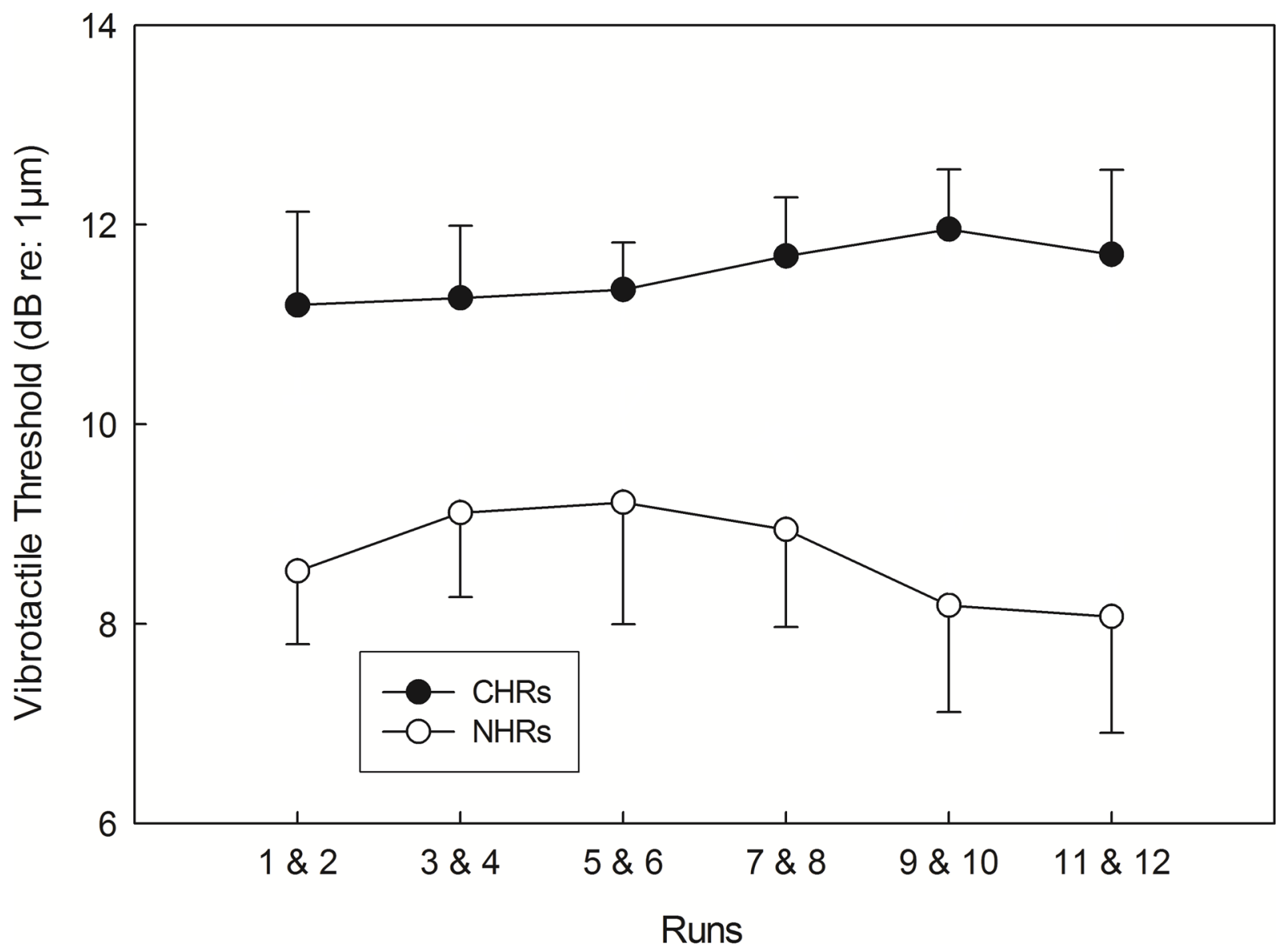

Figure 2.

Effect of pain on vibrotactile thresholds in repeated-gating group. The six measurements of touch gating are shown as a function of the respective runs that comprised each measurement number. Half of the subjects underwent CHRs during even runs and NHRs during odd runs, and for the remaining subjects this order was reversed. Error bars represent +/- 1 SEM. 


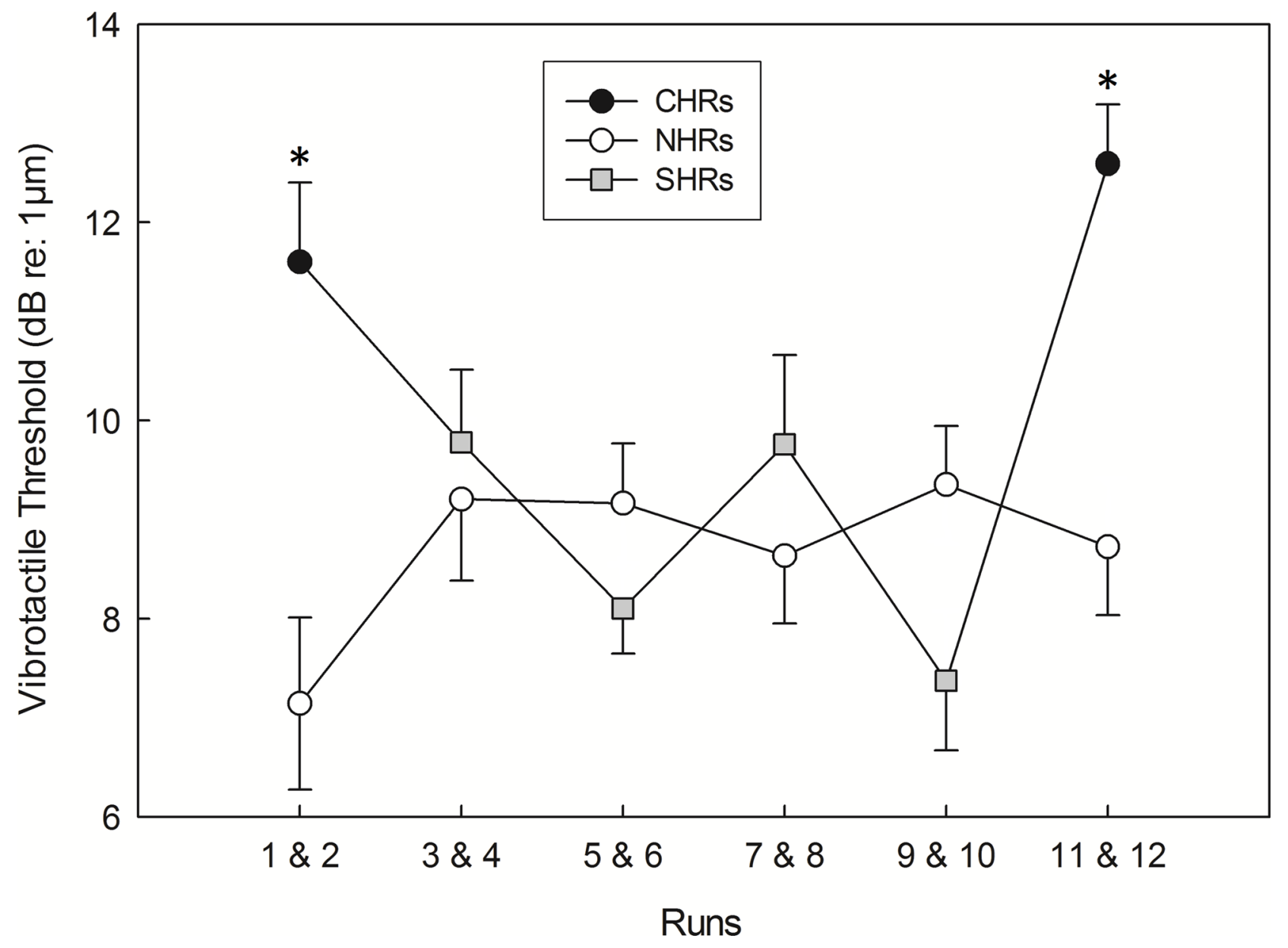

Figure 3.

Effect of pain on vibrotactile thresholds in limited-gating group. Initial (runs 1 and 2) and final (runs 11 and 12) measures of touch gating were assessed in the same manner as in the repeated-gating group. During runs 3-10, heat was never presented during measurements of vibration threshold; instead NHRs alternated with SHRs. Concurrent heat significantly increased vibration thresholds in both the initial and final measures. There was no significant difference between NHR and SHR thresholds. Error bars represent +/- 1 SEM. * indicates $\mathrm{p}<.001$, paired-samples t-test. 


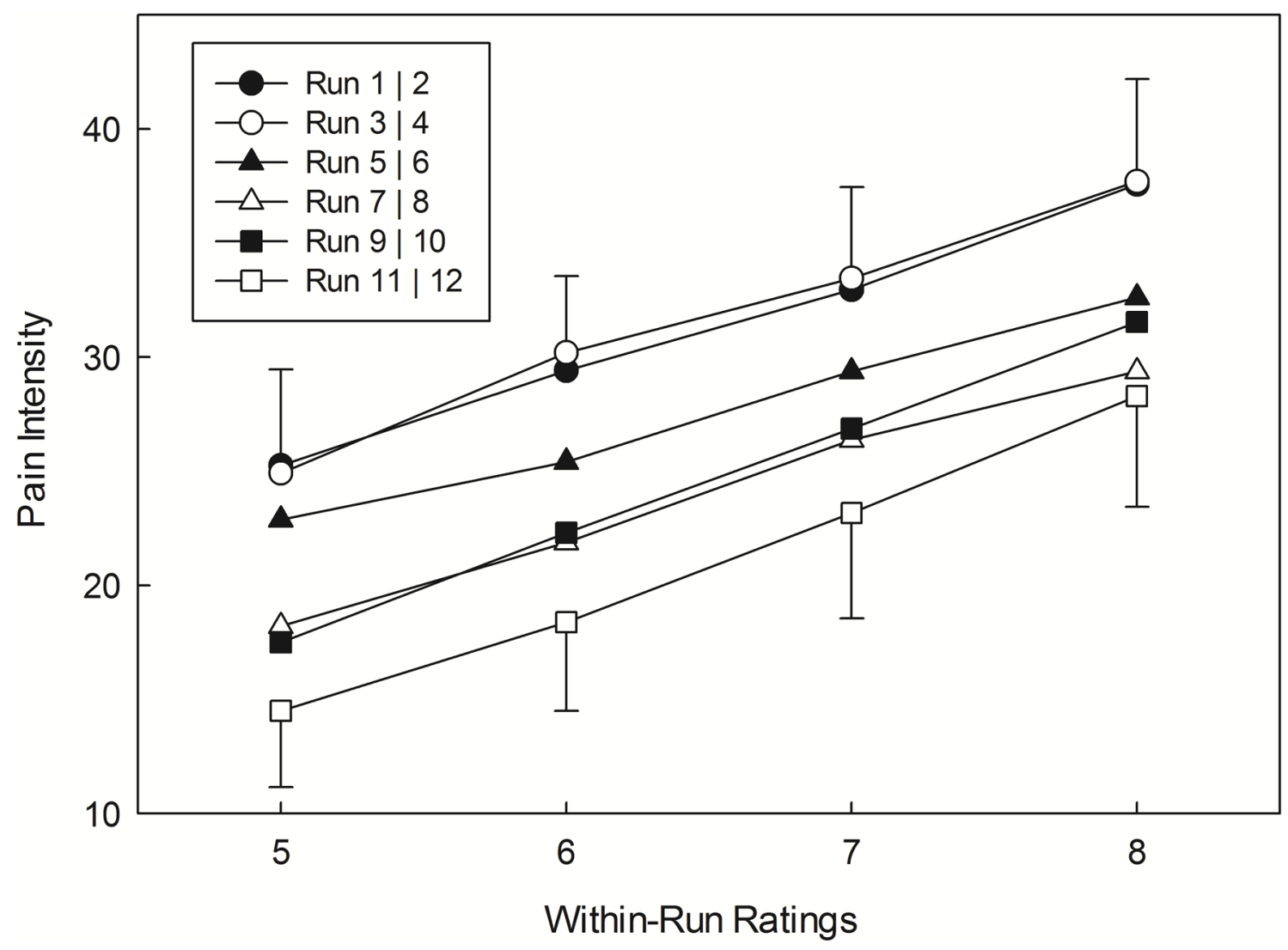

Figure 4.

Perceived pain intensity during six runs containing noxious heat (CHRs and SHRs).

Perceived pain increased significantly within runs while the VLS temperature remained at $45^{\circ} \mathrm{C}$. Error bars representing $+/-1$ SEM are provided for the first and final pain run; variance in the other runs was similar. 


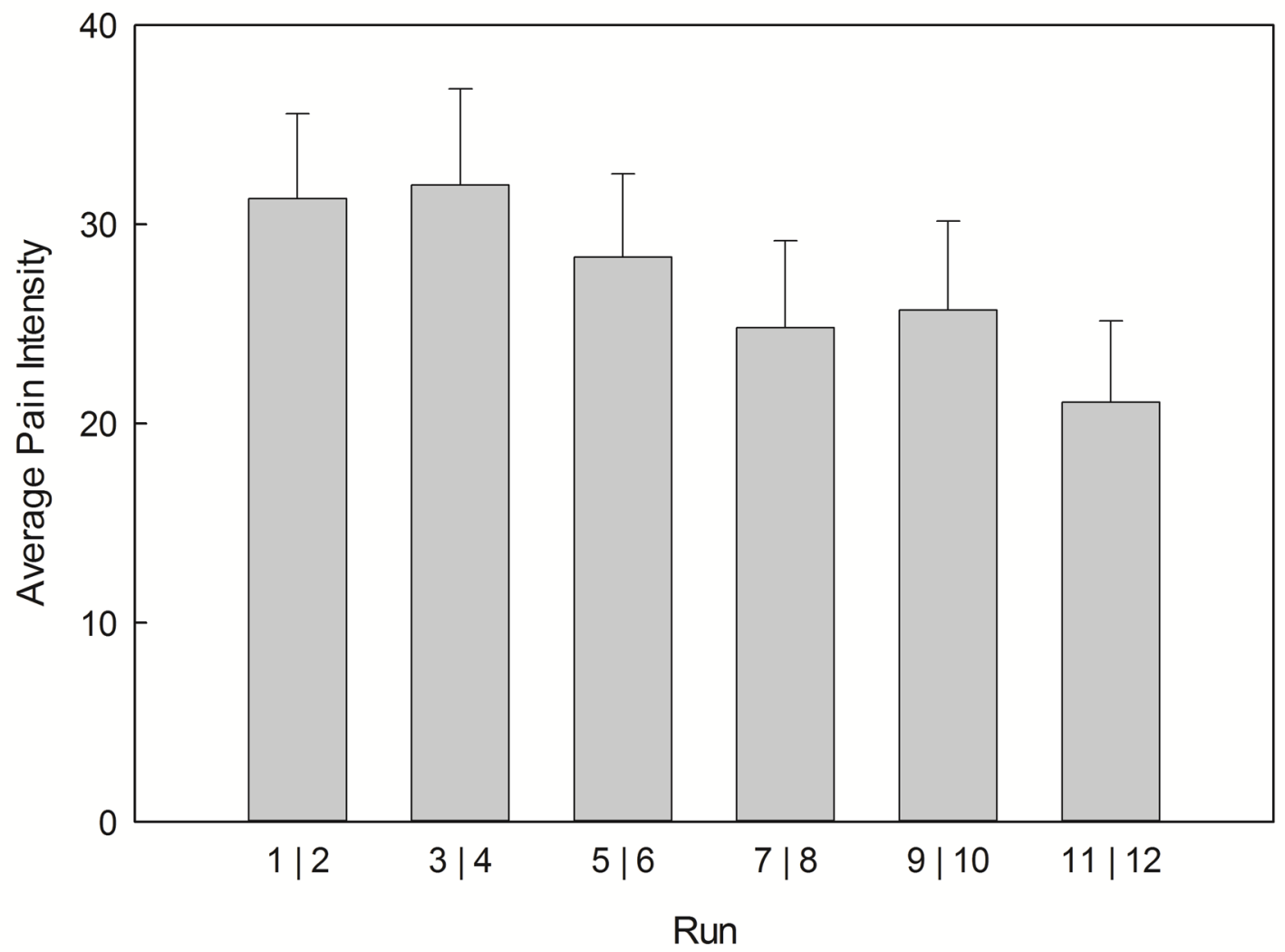

Figure 5.

Pain habituation. Each bar represents the average of ratings given while the VLS temperature was $45^{\circ} \mathrm{C}$ during pain runs (CHRs and SHRs). Since half of the subjects underwent pain runs in even runs and the other half in odd runs, the figure groups the pain ratings from each half of a session together (e.g. run 1 or 2). Significant habituation occurred within and across sessions. Error bars represent + 1 SEM. 


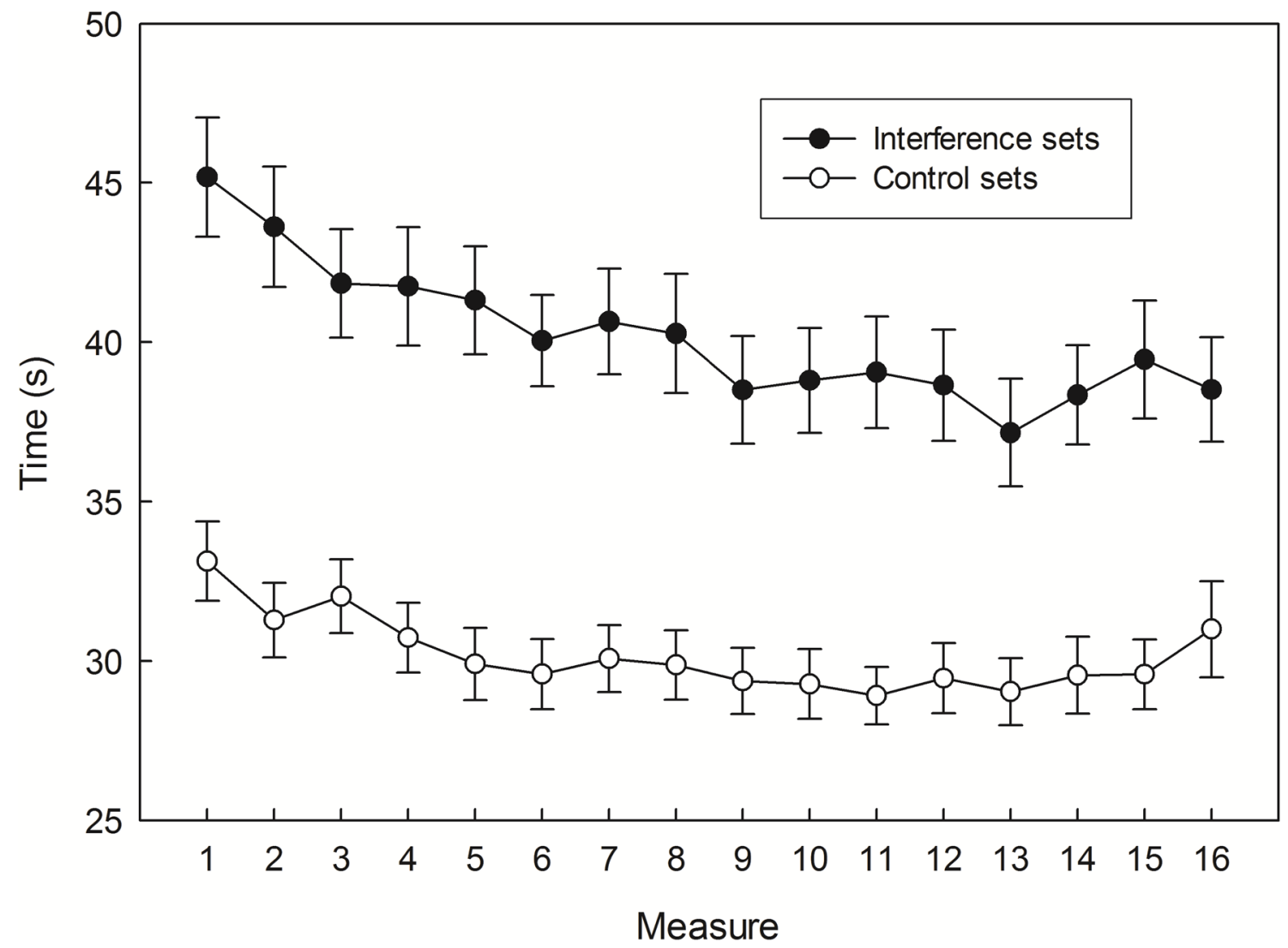

Figure 6.

Stroop interference. Measures 1-4, 5-8, 9-12, and 13-16 took place during Sessions 1, 2, 3, and 4 , respectively. Completion time for interference sets was significantly greater than completion time for control sets for all measures. Error bars represent +/- 1 SEM. 


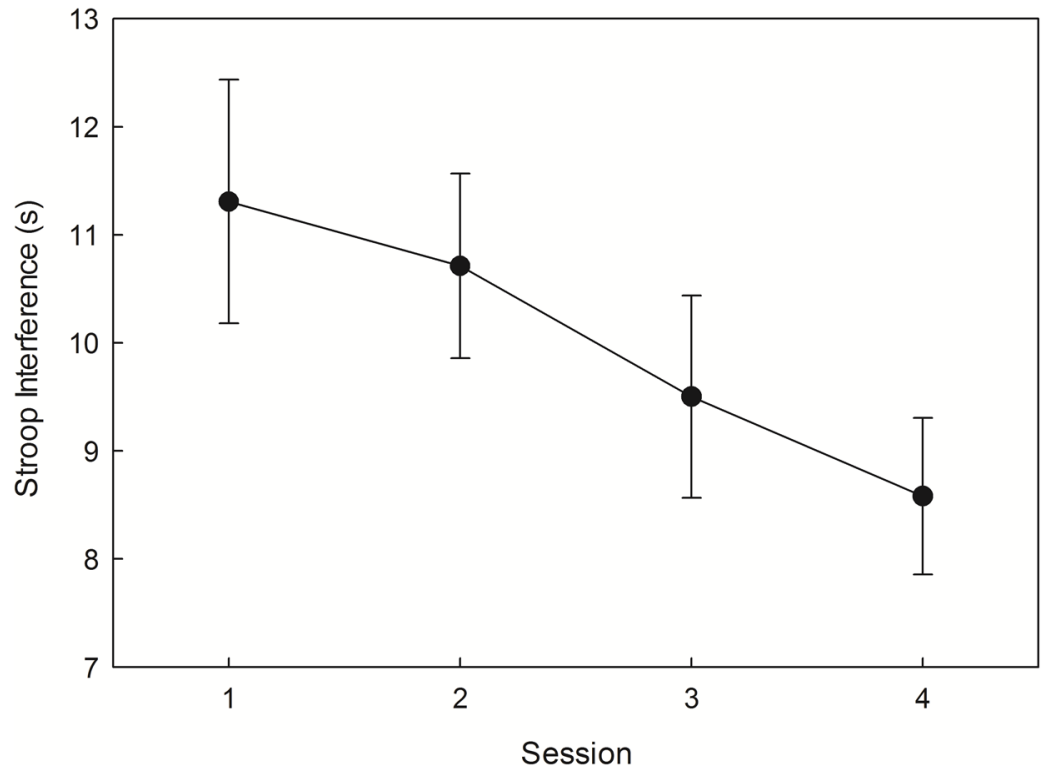

Figure 7.

Stroop interference declined across sessions. Points represent mean Stroop interference (mean of interference sets - mean of control sets) for each session. Error bars represent +/1 SEM. 


\section{Table 1}

Questionnaire scores and comparisons to initial and final measures of touch gating

\begin{tabular}{|c|c|c|c|c|}
\hline & $\begin{array}{c}\text { Session 2 } \\
\text { M (SD) }\end{array}$ & $\begin{array}{c}\text { TG Initial } \\
\mathbf{r}(\boldsymbol{p})\end{array}$ & $\begin{array}{c}\text { Session 4 } \\
\text { M (SD) }\end{array}$ & $\begin{array}{c}\text { TG Final } \\
\mathbf{r}(\boldsymbol{p})\end{array}$ \\
\hline PILL & $18.3(8.9)$ & $.25(.23)$ & $17.3(9.2)$ & $-.20(.35)$ \\
\hline SAI & $35.0(12.9)$ & $.23(.28)$ & $31.7(7.9)$ & $-.25(.24)$ \\
\hline FOPQ & $76.8(20.7)$ & $.18(.40)$ & $79.4(20.6)$ & $-.06(.79)$ \\
\hline CFQ & $45.4(11.4)$ & $.17(.43)$ & $43.8(9.1)$ & $.01(.96)$ \\
\hline Recent Pain & $9.3(9.0)$ & $-.32(.13)$ & $11.8(14.4)$ & $.05(.81)$ \\
\hline Current Pain & $3.2(4.9)$ & $-.15(.47)$ & $2.8(5.4)$ & $-.17(.43)$ \\
\hline
\end{tabular}

Note. $\mathrm{M}=$ mean; $\mathrm{SD}=$ standard deviation; $\mathrm{r}$ = Pearson coefficient for relationship between touch gating (TG) and questionnaires recorded during respective session; $p=p$ value for $\mathrm{r}$; PILL = Pennebaker Inventory of Limbic Languidness; SAI = State Anxiety Inventory; FOPQ = Fear of Pain Questionnaire - III; CFQ = Cognitive Failures Questionnaire; Recent Pain = average pain intensity during past two weeks (0-100 scale); Current Pain = intensity of pain reported at beginning of session (0-100 scale). 